\title{
The presentation of a new inhibitor to prevent enzymatic browning in mushroom, banana, and apple
}

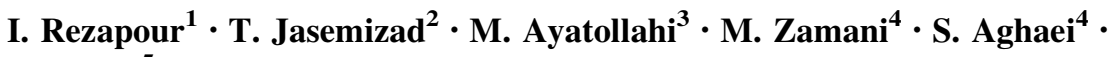 \\ A. Jebali ${ }^{5}$
}

Received: 20 August 2015/Revised: 27 November 2015/Accepted: 14 December 2015/Published online: 7 January 2016

(C) Islamic Azad University (IAU) 2015

\begin{abstract}
The aim of this study was to evaluate a computer-based method to find a new inhibitor for polyphenol oxidase (PPO) in banana, apple, and mushroom. First, the sequence of PPOs was separately obtained from Protein Data Bank, and their homology was investigated. Next, the same structure of their active site was found, and it was interacted with various phenolic and benzoic compounds by a molecular dynamic software. Moreover, the inhibition of enzymatic browning was also investigated at different laboratory conditions. This study showed that histidineleucine-phenylalanine-histidine was in all types of PPOs. Also, molecular dynamic simulation showed that (3S)-2(3,4-dihydroxyphenyl)-3,5,7-chromanetriol (DHPC) is the best compound to interact with PPOs. Based on experimental tests, DHPC had the highest efficacy at $4{ }^{\circ} \mathrm{C}$. The
\end{abstract}

I. Rezapour and T. Jasemizad have contributed equally.

Electronic supplementary material The online version of this article (doi:10.1007/s13762-015-0930-y) contains supplementary material, which is available to authorized users.

A. Jebali

alijebal2011@gmail.com

1 Department of Laboratory Sciences, School of Paramedicine, Shahid Sadoughi University of Medical Sciences, Yazd, Iran

2 Department of Environmental Health Engineering, School of Public Health, Shahid Sadoughi University of Medical Sciences, Yazd, Iran

3 Pharmaceutics Research Center, Faculty of Pharmacy, Kerman University of Medical Sciences, Kerman, Iran

4 Department of Medical Genetics, Faculty of Medicine, Shahid Sadoughi University of Medical Sciences, Yazd, Iran

5 Department of Advanced Medical Sciences and Technologies, School of Paramedicine, Shahid Sadoughi University of Medical Sciences, Yazd, Iran decrease in inhibition of enzymatic browning was seen with the increase in temperature. Also, the decrease in $\mathrm{pH}$ led to increase in enzymatic browning. It could be concluded that DHPC is a good inhibitor for enzymatic browning. It seems that this compound can be used in different fruits and vegetables to inhibit enzymatic browning.

Keywords Browning · Enzyme · Inhibition · Polyphenol oxidase

\section{Introduction}

Peeling, cutting, and crushing lead to changes of physiological and biochemical properties of food products (Moelants et al. 2014; Niemira and Fan 2014). These changes are important causes of loss quality in fruits and vegetables, i.e., appearance, nutritional value, and marketability (Artes and Allende 2014; Rico et al. 2007; Zhang et al. 2015). Theoretically, the enzymatic browning in fruits and vegetables is due to activation of diphenol oxidase, polyphenol oxidase (PPO), catecholase, or tyrosinase (Mishra et al. 2013). The enzyme is found in many plant tissues, especially those that produce brown filaments (Giri 2014; Zhang et al. 2015). PPO catalyzes two basic reactions, including hydroxylation and oxidation. In hydroxylation, monophenols are converted to diphenol. And in oxidation, diphenols are converted to orthoquinones. Ortho-quinones can be polymerized and create the high molecular weight compound, melanin (Bajwa et al. 2015; Corzo-Martınez et al. 2012). Melanin can also react with various amino acids and proteins and leads to brown color. Enzymatic browning causes the deterioration of fruits and vegetables, resulting in large economic losses (Dodd 2014). The browning of injured fruit tissues can cause 


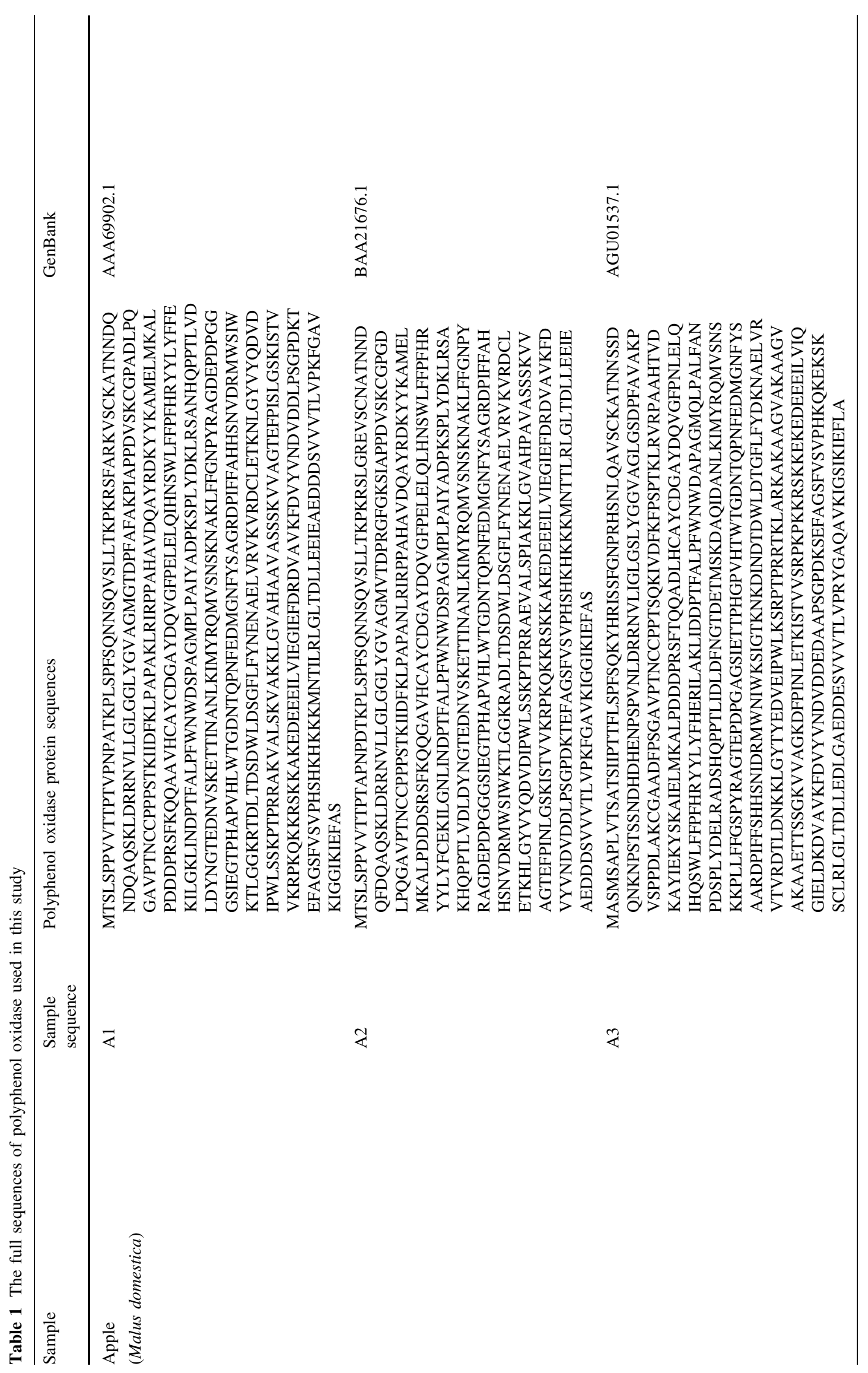




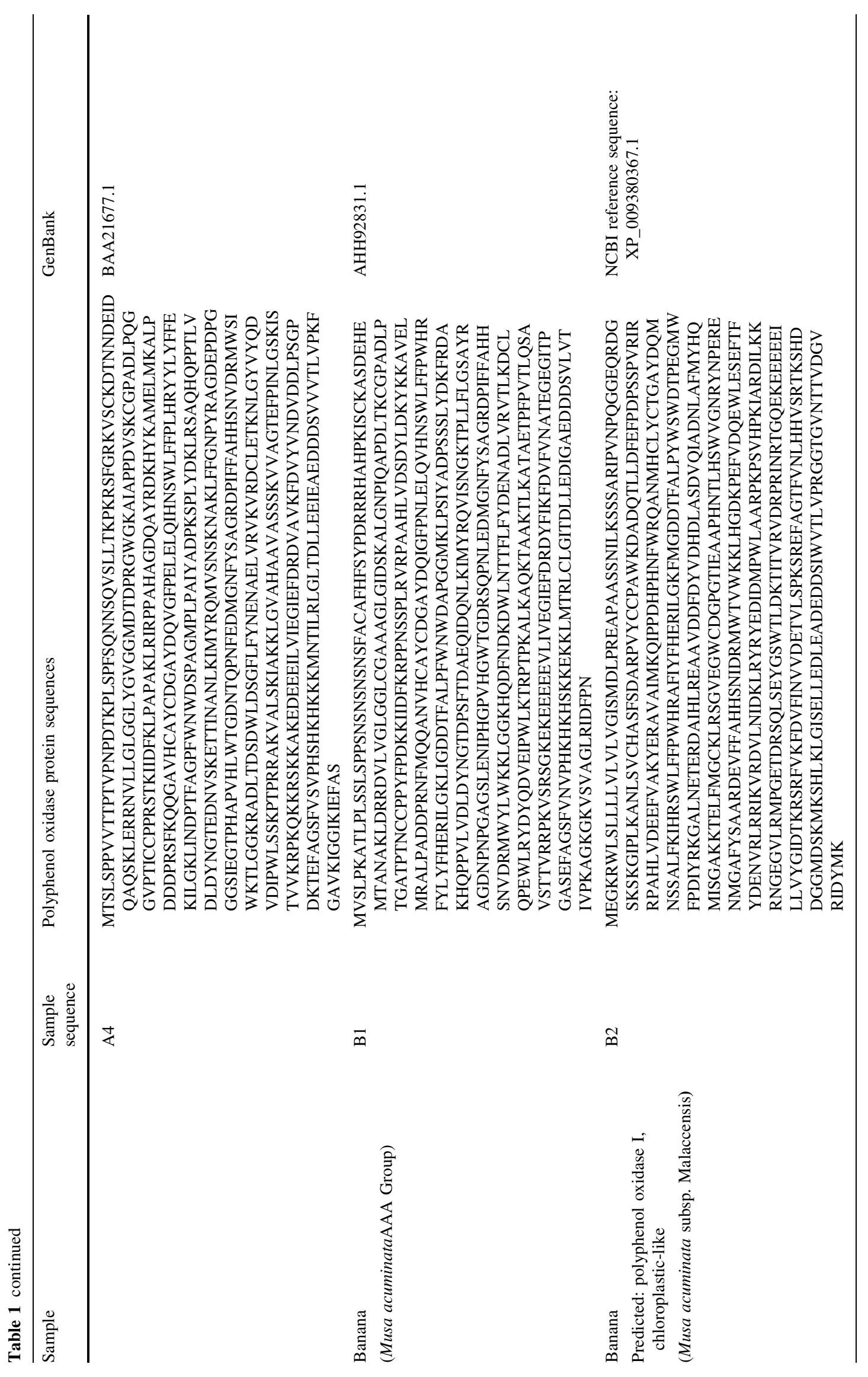




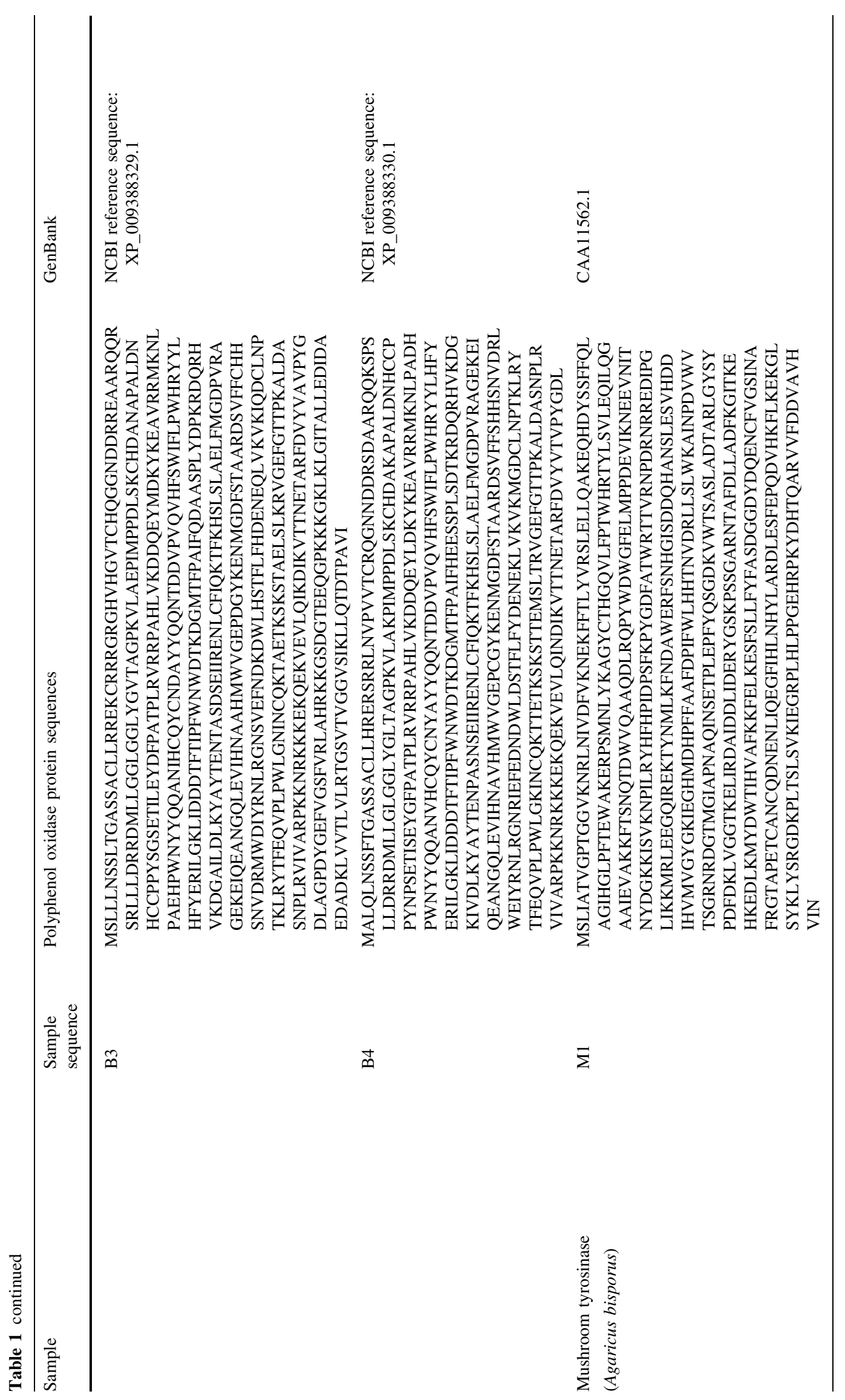




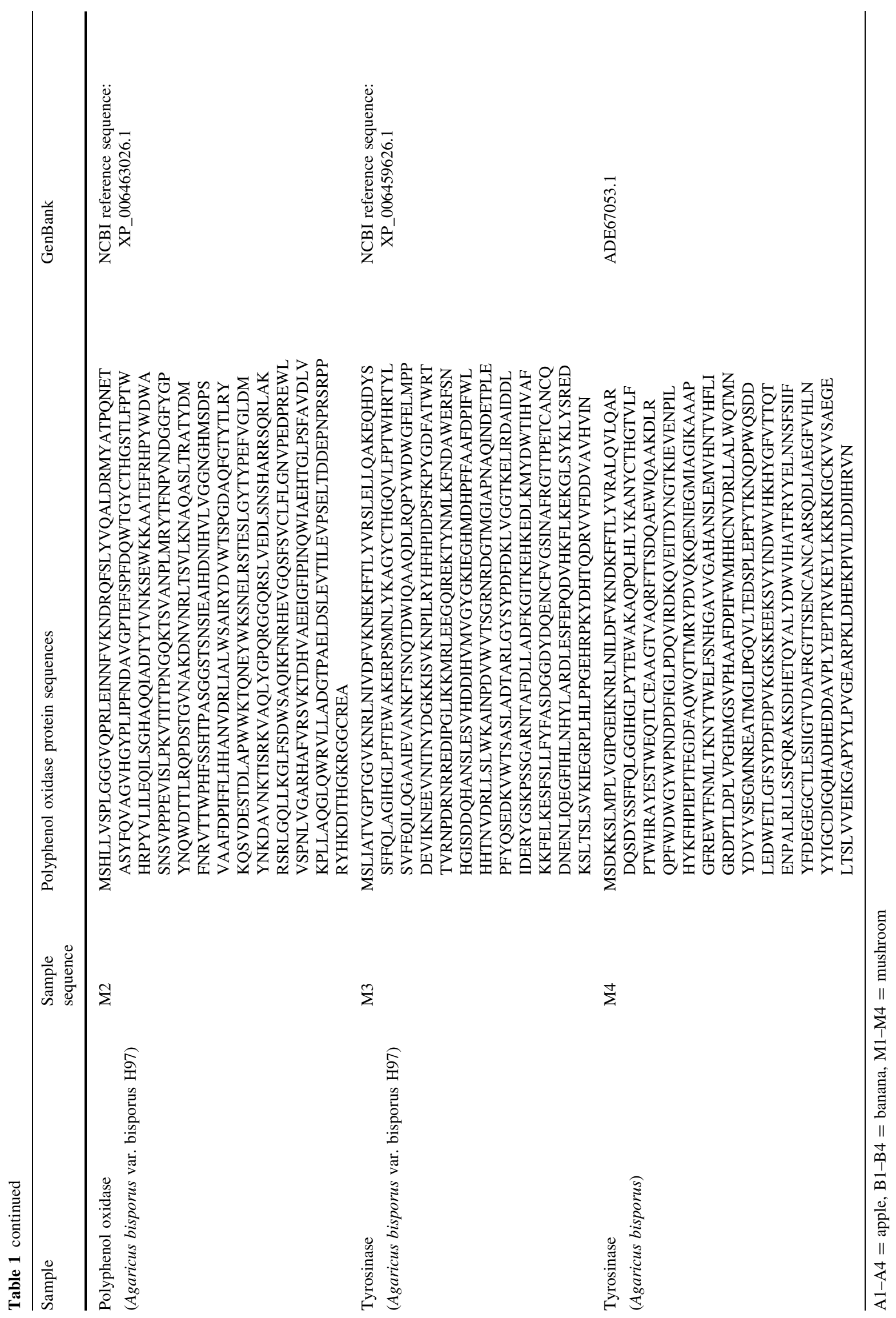


Table 2 The homology between different polyphenol oxidases used in this study

\begin{tabular}{|c|c|}
\hline Enzyme sequence & Identity $\%$ \\
\hline $\mathrm{A} 1-\mathrm{A} 2$ & $95.3 \%$ identity in 593 residues overlap; score: 3008.0 ; gap frequency: $0.0 \%$ \\
\hline $\mathrm{A} 1-\mathrm{A} 3$ & $69.7 \%$ identity in 617 residues overlap; score: 2144.0 ; gap frequency: $4.7 \%$ \\
\hline $\mathrm{A} 1-\mathrm{A} 4$ & $95.8 \%$ identity in 593 residues overlap; score: 3016.0 ; gap frequency: $0.0 \%$ \\
\hline A2-A3 & $67.1 \%$ identity in 617 residues overlap; score: 2064.0; gap frequency: $4.7 \%$ \\
\hline $\mathrm{A} 2-\mathrm{A} 4$ & $94.8 \%$ identity in 593 residues overlap; score: 2994.0 ; gap frequency: $0.0 \%$ \\
\hline A3-A4 & $66.9 \%$ identity in 617 residues overlap; score: 2049.0 ; gap frequency: $4.7 \%$ \\
\hline B1-B2 & $44.3 \%$ identity in 524 residues overlap; score: 1067.0; gap frequency: $5.3 \%$ \\
\hline B1-B3 & $43.8 \%$ identity in 536 residues overlap; score: 1028.0; gap frequency: $4.1 \%$ \\
\hline B1-B4 & $42.4 \%$ identity (67.8\% similar) in 509 aa overlap (19-512:6-503) \\
\hline B2-B3 & $39.6 \%$ identity in 502 residues overlap; score: 844.0 ; gap frequency: $4.6 \%$ \\
\hline B2-B4 & $36.9 \%$ identity (66.7 \% similar) in 496 aa overlap (21-488:26-499) \\
\hline B3-B4 & $87.6 \%$ identity (94.7 \% similar) in 508 aa overlap (1-508:1-504) \\
\hline M1-M2 & $40.2 \%$ identity in 376 residues overlap; score: 719.0 ; gap frequency: $3.2 \%$ \\
\hline M1-M3 & $98.4 \%$ identity in 556 residues overlap; score: 2957.0 ; gap frequency: $0.0 \%$ \\
\hline M1-M4 & $48.8 \%$ identity in 578 residues overlap; score: 1342.0 ; gap frequency: $5.2 \%$ \\
\hline M2-M3 & $39.9 \%$ identity in 376 residues overlap; score: 722.0 ; gap frequency: $3.2 \%$ \\
\hline M2-M4 & $37.0 \%$ identity in 387 residues overlap; score: 563.0 ; gap frequency: $6.2 \%$ \\
\hline M3-M4 & $49.3 \%$ identity in 578 residues overlap; score: 1357.0 ; gap frequency: $5.2 \%$ \\
\hline A1-B1 & $62.2 \%$ identity in 556 residues overlap; score: 1747.0 ; gap frequency: $4.1 \%$ \\
\hline $\mathrm{A} 1-\mathrm{B} 2$ & $42.8 \%$ identity in 484 residues overlap; score: 947.0 ; gap frequency: $3.7 \%$ \\
\hline A1-B3 & $41.5 \%$ identity in 540 residues overlap; score: 945.0 ; gap frequency: $4.8 \%$ \\
\hline A1-B4 & $42.4 \%$ identity in 467 residues overlap; score: 859.0 ; gap frequency: $4.3 \%$ \\
\hline A2-B2 & $41.7 \%$ identity in 484 residues overlap; score: 921.0 ; gap frequency: $3.7 \%$ \\
\hline A2-B3 & $40.3 \%$ identity in 539 residues overlap; score: 910.0 ; gap frequency: $4.5 \%$ \\
\hline A2-B4 & $40.9 \%$ identity in 464 residues overlap; score: 816.0 ; gap frequency: $4.3 \%$ \\
\hline A3-B1 & $59.5 \%$ identity in 555 residues overlap; score: 1697.0 ; gap frequency: $5.2 \%$ \\
\hline A3-B2 & $42.8 \%$ identity in 500 residues overlap; score: 930.0 ; gap frequency: $5.6 \%$ \\
\hline A3-B3 & $41.4 \%$ identity in 551 residues overlap; score: 875.0 ; gap frequency: $7.1 \%$ \\
\hline A3-B4 & $40.7 \%$ identity in 477 residues overlap; score: 807.0 ; gap frequency: $5.5 \%$ \\
\hline A4-B1 & $60.3 \%$ identity in 556 residues overlap; score: 1680.0 ; gap frequency: $4.1 \%$ \\
\hline A4-B2 & $41.9 \%$ identity in 484 residues overlap; score: 921.0 ; gap frequency: $3.7 \%$ \\
\hline A4-B3 & $40.1 \%$ identity in 539 residues overlap; score: 900.0; gap frequency: $4.5 \%$ \\
\hline A4-B4 & $40.6 \%$ identity in 463 residues overlap; score: 804.0 ; gap frequency: $3.9 \%$ \\
\hline A1-M1 & $50.0 \%$ identity in 30 residues overlap; score: 88.0 ; gap frequency: $0.0 \%$ \\
\hline $\mathrm{A} 1-\mathrm{M} 2$ & $54.2 \%$ identity in 24 residues overlap; score: 82.0 ; gap frequency: $0.0 \%$ \\
\hline A1-M3 & $50.0 \%$ identity in 30 residues overlap; score: 88.0 ; gap frequency: $0.0 \%$ \\
\hline A1-M4 & $50.0 \%$ identity in 30 residues overlap; score: 89.0 ; gap frequency: $0.0 \%$ \\
\hline A2-M1 & $50.0 \%$ identity in 30 residues overlap; score: 88.0 ; gap frequency: $0.0 \%$ \\
\hline A2-M2 & $54.2 \%$ identity in 24 residues overlap; score: 82.0 ; gap frequency: $0.0 \%$ \\
\hline A2-M3 & $50.0 \%$ identity in 30 residues overlap; score: 88.0 ; gap frequency: $0.0 \%$ \\
\hline A2-M4 & $50.0 \%$ identity in 30 residues overlap; score: 89.0 ; gap frequency: $0.0 \%$ \\
\hline A3-M1 & $38.1 \%$ identity in 63 residues overlap; score: 91.0 ; gap frequency: $4.8 \%$ \\
\hline A3-M2 & $58.3 \%$ identity in 24 residues overlap; score: 84.0 ; gap frequency: $0.0 \%$ \\
\hline A3-M3 & $38.1 \%$ identity in 63 residues overlap; score: 91.0 ; gap frequency: $4.8 \%$ \\
\hline A3-M4 & $46.7 \%$ identity in 30 residues overlap; score: 84.0 ; gap frequency: $0.0 \%$ \\
\hline A4-M1 & $50.0 \%$ identity in 30 residues overlap; score: 88.0 ; gap frequency: $0.0 \%$ \\
\hline A4-M2 & $54.2 \%$ identity in 24 residues overlap; score: 82.0 ; gap frequency: $0.0 \%$ \\
\hline A4-M3 & $50.0 \%$ identity in 30 residues overlap; score: 88.0 ; gap frequency: $0.0 \%$ \\
\hline A4-M4 & $50.0 \%$ identity in 30 residues overlap; score: 89.0 ; gap frequency: $0.0 \%$ \\
\hline B1-M1 & $50.0 \%$ identity in 30 residues overlap; score: 83.0 ; gap frequency: $0.0 \%$ \\
\hline
\end{tabular}


Table 2 continued

\begin{tabular}{ll}
\hline Enzyme sequence & Identity \% \\
\hline B1-M2 & $58.3 \%$ identity in 24 residues overlap; score: 80.0 ; gap frequency: $0.0 \%$ \\
B1-M3 & $50.0 \%$ identity in 30 residues overlap; score: 83.0; gap frequency: $0.0 \%$ \\
B1-M4 & $50.0 \%$ identity in 30 residues overlap; score: 82.0 ; gap frequency: $0.0 \%$ \\
B2-M1 & $35.3 \%$ identity in 51 residues overlap; score: 86.0 ; gap frequency: $3.9 \%$ \\
B2-M2 & $44.4 \%$ identity in 27 residues overlap; score: 76.0 ; gap frequency: $0.0 \%$ \\
B2-M3 & $35.3 \%$ identity in 51 residues overlap; score: 86.0 ; gap frequency: $3.9 \%$ \\
B2-M4 & $38.2 \%$ identity in 34 residues overlap; score: 80.0 ; gap frequency: $0.0 \%$ \\
B3-M1 & $35.0 \%$ identity in 60 residues overlap; score: 71.0 ; gap frequency: $3.3 \%$ \\
B3-M2 & $38.2 \%$ identity in 55 residues overlap; score: 82.0; gap frequency: $7.3 \%$ \\
B3-M3 & $35.0 \%$ identity in 60 residues overlap; score: 71.0 ; gap frequency: $3.3 \%$ \\
B3-M4 & $32.7 \%$ identity in 55 residues overlap; score: 75.0 ; gap frequency: $7.3 \%$ \\
B4-M1 & $36.7 \%$ identity in 60 residues overlap; score: 75.0 ; gap frequency: $3.3 \%$ \\
B4-M2 & $40.0 \%$ identity in 55 residues overlap; score: 88.0 ; gap frequency: $7.3 \%$ \\
B4-M3 & $36.7 \%$ identity in 60 residues overlap; score: 75.0 ; gap frequency: $3.3 \%$ \\
B4-M4 & $36.4 \%$ identity in 55 residues overlap; score: 80.0 ; gap frequency: $7.3 \%$ \\
\hline
\end{tabular}

undesirable quality changes during handling, processing, and storage (Ali et al. 2015; Quevedo et al. 2014a, b). To prevent PPO activity in fruits and vegetables, many efforts have been previously done. One of them is the use of reducers which revive o-quinones precursors and convert them into non-colored compounds (Wu 2014; Zhou et al. 2015). The inhibitors of PPO are classified into two categories, including competitive and non-competitive. Importantly, competitive inhibitors interact with the copper site, and non-competitive inhibitors interact with the phenolic site (Ackaah-Gyasi et al. 2015; Boeckx et al. 2015). For example, bisulfite is a competitive inhibitor, and L-cysteine is a non-competitive inhibitor (Ali et al. 2014; Saeidian 2014).

To find an inhibitor for a specific enzyme, a computerbased method $(\mathrm{CBM})$ has been introduced (JiménezAtiénzar et al. 2004; Ma et al. 2014). Although researchers have been studied on different inhibitors (Altunkaya and Gökmen 2008; Kuijpers and Vincken 2013), most of them are toxic and can change texture and taste of fruits. Here, a CBM was used to find a new inhibitor for PPO in two types of fruits (banana and apple) and one type of mushroom. Moreover, the efficacy of inhibitor was checked by some experiments. This article carried out in the Pajoohesh Medical Lab, Yazd, in 2015.

\section{Materials and methods}

\section{Materials}

(3S)-2-(3,4-dihydroxyphenyl)-3,5,7-chromanetriol (DHPC) was purchased from Merck, Germany. Apple, banana, and
Table 3 The average homology between different polyphenol oxidases

\begin{tabular}{ll}
\hline Sample & Mean of identity (\%) \\
\hline Apple & 81.6 \\
Banana & 49.1 \\
mushroom & 52.6 \\
Apple-Banana & 65.35 \\
Apple-mushroom & 73.47 \\
Banana-mushroom & 50.85 \\
\hline
\end{tabular}

mushroom were provided from different shops of Yazd, Iran.

\section{Simulation study}

PPO sequences of apple, banana, and mushroom were separately obtained from Protein Data Bank (PDB), http:// www.rcsb.org/pdb (Table 1). In order to check homology of these sequences, an online software was used, http:// www.isb-sib.ch. Then, the place of all histidines $(\mathrm{H})$ and all phenyl alanines (F) was highlighted. Then, the quantity of HH, FF, HF, FH, H1-20H, F1-20F, H1-20F, and F1-20H was quantified. In the next level, the best sequence was selected, based on its frequency. Then, this sequence was interacted with various phenolic and benzoic compounds by a molecular dynamic (MD) software, Ascalaph Designer. Finally, the average of intermolecular energy was calculated for each compound. 
Table 4 The quantity of different sequences, containing histidines (H) and phenyl alanines (F)

\begin{tabular}{|c|c|c|c|c|c|c|c|}
\hline Sequences & Quantity & Sequences & Quantity & Sequences & Quantity & Sequences & Quantity \\
\hline $\mathrm{FF}$ & 12 & $\mathrm{FH}$ & 10 & $\mathrm{HF}$ & 7 & $\mathrm{HH}$ & 12 \\
\hline $\mathrm{F} 1 \mathrm{~F}$ & 11 & $\mathrm{~F} 1 \mathrm{H}$ & 12 & $\mathrm{H} 1 \mathrm{~F}$ & 5 & $\mathrm{H} 1 \mathrm{H}$ & 9 \\
\hline $\mathrm{F} 2 \mathrm{~F}$ & 8 & $\mathrm{~F} 2 \mathrm{H}$ & 12 & $\mathrm{H} 2 \mathrm{~F}$ & 6 & $\mathrm{H} 2 \mathrm{H}$ & 3 \\
\hline $\mathrm{F} 3 \mathrm{~F}$ & 12 & $\mathrm{~F} 3 \mathrm{H}$ & 12 & $\mathrm{H} 3 \mathrm{~F}$ & 2 & $\mathrm{H} 3 \mathrm{H}$ & 12 \\
\hline $\mathrm{F} 4 \mathrm{~F}$ & 5 & $\mathrm{~F} 4 \mathrm{H}$ & 9 & $\mathrm{H} 4 \mathrm{~F}$ & 12 & $\mathrm{H} 4 \mathrm{H}$ & 3 \\
\hline $\mathrm{F} 5 \mathrm{~F}$ & 9 & F5H & 4 & $\mathrm{H} 5 \mathrm{~F}$ & 12 & $\mathrm{H} 5 \mathrm{H}$ & 4 \\
\hline F6F & 8 & F6H & 11 & $\mathrm{H} 6 \mathrm{~F}$ & 4 & $\mathrm{H} 6 \mathrm{H}$ & 6 \\
\hline F7F & 8 & $\mathrm{~F} 7 \mathrm{H}$ & 7 & $\mathrm{H} 7 \mathrm{~F}$ & 2 & $\mathrm{H} 7 \mathrm{H}$ & 4 \\
\hline $\mathrm{F} 8 \mathrm{~F}$ & 10 & $\mathrm{~F} 8 \mathrm{H}$ & 4 & $\mathrm{H} 8 \mathrm{~F}$ & 4 & $\mathrm{H} 8 \mathrm{H}$ & 12 \\
\hline F9F & 10 & $\mathrm{~F} 9 \mathrm{H}$ & 3 & $\mathrm{H} 9 \mathrm{~F}$ & 1 & $\mathrm{H} 9 \mathrm{H}$ & 6 \\
\hline $\mathrm{F} 10 \mathrm{~F}$ & 4 & $\mathrm{~F} 10 \mathrm{H}$ & 0 & $\mathrm{H} 10 \mathrm{~F}$ & 4 & $\mathrm{H} 10 \mathrm{H}$ & 5 \\
\hline $\mathrm{F} 11 \mathrm{~F}$ & 4 & $\mathrm{~F} 11 \mathrm{H}$ & 3 & $\mathrm{H} 11 \mathrm{~F}$ & 1 & $\mathrm{H} 11 \mathrm{H}$ & 4 \\
\hline $\mathrm{F} 12 \mathrm{~F}$ & 8 & $\mathrm{~F} 12 \mathrm{H}$ & 1 & $\mathrm{H} 12 \mathrm{~F}$ & 5 & $\mathrm{H} 12 \mathrm{H}$ & 2 \\
\hline $\mathrm{F} 13 \mathrm{~F}$ & 8 & $\mathrm{~F} 13 \mathrm{H}$ & 1 & $\mathrm{H} 13 \mathrm{~F}$ & 1 & $\mathrm{H} 13 \mathrm{H}$ & 2 \\
\hline $\mathrm{F} 14 \mathrm{~F}$ & 4 & $\mathrm{~F} 14 \mathrm{H}$ & 2 & $\mathrm{H} 14 \mathrm{~F}$ & 3 & $\mathrm{H} 14 \mathrm{H}$ & 4 \\
\hline $\mathrm{F} 15 \mathrm{~F}$ & 4 & $\mathrm{~F} 15 \mathrm{H}$ & 3 & $\mathrm{H} 15 \mathrm{~F}$ & 2 & $\mathrm{H} 15 \mathrm{H}$ & 2 \\
\hline $\mathrm{F} 16 \mathrm{~F}$ & 3 & $\mathrm{~F} 16 \mathrm{H}$ & 2 & $\mathrm{H} 16 \mathrm{~F}$ & 3 & $\mathrm{H} 16 \mathrm{H}$ & 3 \\
\hline $\mathrm{F} 17 \mathrm{~F}$ & 1 & $\mathrm{~F} 17 \mathrm{H}$ & 1 & $\mathrm{H} 17 \mathrm{~F}$ & 1 & $\mathrm{H} 17 \mathrm{H}$ & 0 \\
\hline $\mathrm{F} 18 \mathrm{~F}$ & 7 & $\mathrm{~F} 18 \mathrm{H}$ & 0 & $\mathrm{H} 18 \mathrm{~F}$ & 1 & $\mathrm{H} 18 \mathrm{H}$ & 0 \\
\hline $\mathrm{F} 19 \mathrm{~F}$ & 6 & $\mathrm{~F} 19 \mathrm{H}$ & 2 & $\mathrm{H} 19 \mathrm{~F}$ & 1 & $\mathrm{H} 19 \mathrm{H}$ & 1 \\
\hline $\mathrm{F} 20 \mathrm{~F}$ & 3 & $\mathrm{~F} 20 \mathrm{H}$ & 0 & $\mathrm{H} 20 \mathrm{~F}$ & 0 & $\mathrm{H} 20 \mathrm{H}$ & 5 \\
\hline
\end{tabular}

Table 5 The selected sequences, containing histidines $(\mathrm{H})$ and phenyl alanines $(\mathrm{F})$

\begin{tabular}{|c|c|c|c|c|c|c|c|c|}
\hline & $\mathrm{H} 3 \mathrm{H}$ & $\mathrm{H} 8 \mathrm{H}$ & $\mathrm{H} 4 \mathrm{~F}$ & $\mathrm{H} 5 \mathrm{~F}$ & $\mathrm{~F} 1 \mathrm{H}$ & $\mathrm{F} 2 \mathrm{H}$ & $\mathrm{F} 3 \mathrm{H}$ & $\mathrm{F} 3 \mathrm{~F}$ \\
\hline A1 & $\begin{array}{l}\text { HAPVH } \\
\text { HSHKH }\end{array}$ & HNSWLFFPFH & HNSWLF & HRYYLYF & FAH & $\begin{array}{l}\text { FPFH } \\
\text { FFAH } \\
\text { FAHH }\end{array}$ & $\begin{array}{l}\text { FFPFH } \\
\text { FFAHH }\end{array}$ & $\begin{array}{l}\text { FALPF } \\
\text { FAGSF }\end{array}$ \\
\hline $\mathrm{A} 2$ & $\begin{array}{l}\text { HAPVH } \\
\text { HSHKH }\end{array}$ & HNSWLFFPFH & HNSWLF & HRYYLYF & FAH & $\begin{array}{l}\text { FPFH } \\
\text { FFAH } \\
\text { FAHH }\end{array}$ & $\begin{array}{l}\text { FFPFH } \\
\text { FFAHH }\end{array}$ & $\begin{array}{l}\text { FALPF } \\
\text { FAGSF }\end{array}$ \\
\hline A3 & HGPVH & HQSWLFFPFH & $\begin{array}{l}\text { HRISSF } \\
\text { HQSWLF }\end{array}$ & HRYYLYF & FSH & $\begin{array}{l}\text { FSHH } \\
\text { FPFH } \\
\text { FFSH }\end{array}$ & $\begin{array}{l}\text { FFPFH } \\
\text { FFSHH }\end{array}$ & $\begin{array}{l}\text { FLSPF } \\
\text { FALPF } \\
\text { FAGSF }\end{array}$ \\
\hline A4 & $\begin{array}{l}\text { HSHKH } \\
\text { HAPVH }\end{array}$ & HNSWLFFPLH & HNSWLF & HRYYLYF & FAH & $\begin{array}{l}\text { FAHH } \\
\text { FPLH } \\
\text { FFAH }\end{array}$ & $\begin{array}{l}\text { FFAHH } \\
\text { FFPLH }\end{array}$ & $\begin{array}{l}\text { FAGP } \\
\text { FAGSF }\end{array}$ \\
\hline B1 & $\begin{array}{l}\text { HGPVH } \\
\text { HKHKH }\end{array}$ & $\begin{array}{l}\text { HFSYPDRRRH } \\
\text { HNSWLFFPWH }\end{array}$ & HNSWLF & HNSWLFF & FAH & $\begin{array}{l}\text { FAHH } \\
\text { FPWH } \\
\text { FFAH }\end{array}$ & $\begin{array}{l}\text { FFPWH } \\
\text { FFAHH }\end{array}$ & $\begin{array}{l}\text { FACAF } \\
\text { FAGSF } \\
\text { FYLYF } \\
\text { FALPF }\end{array}$ \\
\hline B2 & HNTLH & $\begin{array}{l}\text { HRSWLFFPWH } \\
\text { HNFWRQANMH }\end{array}$ & HRSWLF & $\begin{array}{l}\text { HRSWLFF } \\
\text { HGDKPEF }\end{array}$ & FAH & $\begin{array}{l}\text { FKIH } \\
\text { FPWH } \\
\text { FMYH } \\
\text { FAHH } \\
\text { FFAH }\end{array}$ & $\begin{array}{l}\text { FFPWH } \\
\text { FIYFH } \\
\text { FFAHH } \\
\text { FVNLH }\end{array}$ & FAGTF \\
\hline
\end{tabular}


Table 5 continued

\begin{tabular}{|c|c|c|c|c|c|c|c|c|}
\hline & $\mathrm{H} 3 \mathrm{H}$ & $\mathrm{H} 8 \mathrm{H}$ & $\mathrm{H} 4 \mathrm{~F}$ & $\mathrm{H} 5 \mathrm{~F}$ & $\mathrm{~F} 1 \mathrm{H}$ & $\mathrm{F} 2 \mathrm{H}$ & $\mathrm{F} 3 \mathrm{H}$ & F3F \\
\hline B3 & HNAAH & HFSWIFLPWH & $\begin{array}{l}\text { HFSWIF } \\
\text { HSTFLF }\end{array}$ & HRYYLHF & $\begin{array}{l}\text { FKH } \\
\text { FCH }\end{array}$ & $\begin{array}{l}\text { FCHH } \\
\text { FLFH } \\
\text { FFCH }\end{array}$ & $\begin{array}{l}\text { FLPWH } \\
\text { FFCHH }\end{array}$ & $\begin{array}{l}\text { FTIPF } \\
\text { FPAIF } \\
\text { FSWIF } \\
\text { FVGSF }\end{array}$ \\
\hline B4 & HNAVH & HFSWIFLPWH & HFSWIF & HRYYLHF & $\begin{array}{l}\text { FKH } \\
\text { FSH }\end{array}$ & $\begin{array}{l}\text { FSHH } \\
\text { FFSH }\end{array}$ & $\begin{array}{l}\text { FLPWH } \\
\text { FFSHH }\end{array}$ & $\begin{array}{l}\text { FTIPF } \\
\text { FPAIF } \\
\text { FSWIF }\end{array}$ \\
\hline M1 & HDDIH & HGQVLFPTWH & $\begin{array}{l}\text { HGQVLF } \\
\text { HDYSSF }\end{array}$ & $\begin{array}{l}\text { HPIDPSF } \\
\text { HDYSSFF }\end{array}$ & FIH & $\begin{array}{l}\text { FWLH } \\
\text { FSNH }\end{array}$ & $\begin{array}{l}\text { FPTWH } \\
\text { FWLHH }\end{array}$ & $\begin{array}{l}\text { FSLLF } \\
\text { FFAAF } \\
\text { FDPIF }\end{array}$ \\
\hline M2 & $\begin{array}{l}\text { HFSSH } \\
\text { HDNIH }\end{array}$ & HGSTLFPTWH & HGSTLF & $\begin{array}{l}\text { HEVGQSF } \\
\text { HTGLPSF }\end{array}$ & $\begin{array}{l}\text { FRH } \\
\text { FLH }\end{array}$ & $\begin{array}{l}\text { FSSH } \\
\text { FLHH } \\
\text { FFLH }\end{array}$ & $\begin{array}{l}\text { FPTWH } \\
\text { FFLHH }\end{array}$ & FDPIF \\
\hline M3 & HDDIH & HGQVLFPTWH & $\begin{array}{l}\text { HGQVLF } \\
\text { HDYSSF }\end{array}$ & $\begin{array}{l}\text { HDYSSFF } \\
\text { HVAFKKF } \\
\text { HPIDPSF }\end{array}$ & FIH & $\begin{array}{l}\text { FWLH } \\
\text { FSNH }\end{array}$ & $\begin{array}{l}\text { FPTWH } \\
\text { FWLHH }\end{array}$ & $\begin{array}{l}\text { FFAAF } \\
\text { FDPIF } \\
\text { FSLLF }\end{array}$ \\
\hline M4 & $\begin{array}{l}\text { HYKFH } \\
\text { HNTVH }\end{array}$ & $\begin{array}{l}\text { HLYKANYCTH } \\
\text { HGTVLFPTWH }\end{array}$ & $\begin{array}{l}\text { HGTVLF } \\
\text { HNTVHF } \\
\text { HKHYGF }\end{array}$ & HPIEPTF & FVH & $\begin{array}{l}\text { FSNH } \\
\text { FWMH }\end{array}$ & $\begin{array}{l}\text { FWMHH } \\
\text { FPTWH }\end{array}$ & $\begin{array}{l}\text { FSIIF } \\
\text { FEGDF } \\
\text { FDPIF }\end{array}$ \\
\hline
\end{tabular}

Table 6 The average of intermolecular energy and number of hydrogen bonding between HLFH and some phenolic and benzoic compounds

\begin{tabular}{|c|c|c|}
\hline & Intermolecular energy & No. of hydrogen bonding \\
\hline$(+)$-Epicatechin & -0.05 & 3 \\
\hline (3S)-2-(3,4-dihydroxyphenyl)-3,5,7-chromanetriol & -7.37 & 3 \\
\hline [3,4-Dihydroxy(2H3)phenyl](2H2)acetic acid & -0.05 & 2 \\
\hline [3,4-Dihydroxy $(5-2 \mathrm{H})$ phenyl]acetic acid & -0.03 & 3 \\
\hline$\{4-[(Z)$-hydroxy(oxonio)methyl]phenyl $\}$ oxonium & -0.00 & 3 \\
\hline 2,3-Dihydroxybenzoate & -0.04 & 3 \\
\hline 2,3-Dihydroxybenzoic acid & -1.80 & 1 \\
\hline 2-Hydroxy(1-14C)benzoic acid & -1.76 & 2 \\
\hline 2-Hydroxy(2H4)benzoic acid & -0.40 & 3 \\
\hline 2-Hydroxy(carboxy-11C)benzoic acid & -0.45 & 2 \\
\hline $3,4,5$-Trihydroxy $(1,3,5-13 \mathrm{C} 3)$ benzoic acid & -0.11 & 3 \\
\hline $3,4,5$-Trihydroxy $(2 \mathrm{H} 2)$ benzoic acid & -0.77 & 2 \\
\hline 3,4,5-Trihydroxybenzoic acid & -0.01 & 3 \\
\hline 3,4-Dihydroxy $(2-2 \mathrm{H})$ benzoic acid & -0.09 & 2 \\
\hline 3,4-Dihydroxy $(5-2 \mathrm{H})$ benzoic acid & -0.32 & 2 \\
\hline 3,4-Dihydroxy $(6-2 \mathrm{H})$ benzoic acid & -0.08 & 3 \\
\hline 3,4-Dihydroxybenzeneacetate & -2.48 & 3 \\
\hline 3,4-Dihydroxybenzoate & -0.97 & 2 \\
\hline 3,4-Dihydroxybenzoic acid & -0.75 & 2 \\
\hline 3,4-Dihydroxyphenylacetic acid & -0.02 & 1 \\
\hline 3-Hydroxybenzoate & -0.94 & 3 \\
\hline 3-Hydroxybenzoic acid & -0.02 & 2 \\
\hline 3-Hydroxybenzoic acid & -1.57 & 2 \\
\hline 4- $(2 \mathrm{H} 3)$ methyl $(2 \mathrm{H} 3)$ benzene-1.2- $(2 \mathrm{H} 2)$ diol & -0.05 & 2 \\
\hline
\end{tabular}


Table 6 continued

\begin{tabular}{|c|c|c|}
\hline & Intermolecular energy & No. of hydrogen bonding \\
\hline 4-(2H3)methyl-1.2-benzenediol & -0.01 & 3 \\
\hline 4-Hydroxy(2H4)benzoic acid & -1.71 & 3 \\
\hline 4-Hydroxy(carboxy-11C)benzoic acid & -0.10 & 2 \\
\hline 4-Hydroxy(carboxy-13C)benzoic acid & -0.53 & 3 \\
\hline 4-Hydroxy(carboxy-13C)benzoic acid1 & -0.02 & 3 \\
\hline 4-Hydroxy(carboxy-14C)benzoic acid & -0.27 & 2 \\
\hline 4-Hydroxybenzoate & -1.29 & 2 \\
\hline 4-Hydroxybenzoic acid & -0.48 & 2 \\
\hline 4-Methyl-1,2-(2H3)benzenediol & -0.00 & 2 \\
\hline 4-Methyl-1,2-(3H3)benzenediol & -0.26 & 2 \\
\hline 4-Methylcatechol & -0.01 & 2 \\
\hline D-(+)-Catechin & -0.07 & 2 \\
\hline DL-CATECHIN & -0.07 & 3 \\
\hline Gallate & -0.22 & 1 \\
\hline Gallic acid & -0.28 & 3 \\
\hline Guaiacol & -2.54 & 3 \\
\hline$o$-Hydroxybenzoic acid & -0.00 & 3 \\
\hline$p$-Hydroxybenzoic acid & -0.03 & 2 \\
\hline Protocatechuic acid & -0.14 & 2 \\
\hline Pyrogallol & -0.01 & 2 \\
\hline Salicylate & -1.53 & 2 \\
\hline Salicylic acid & -0.73 & 2 \\
\hline
\end{tabular}

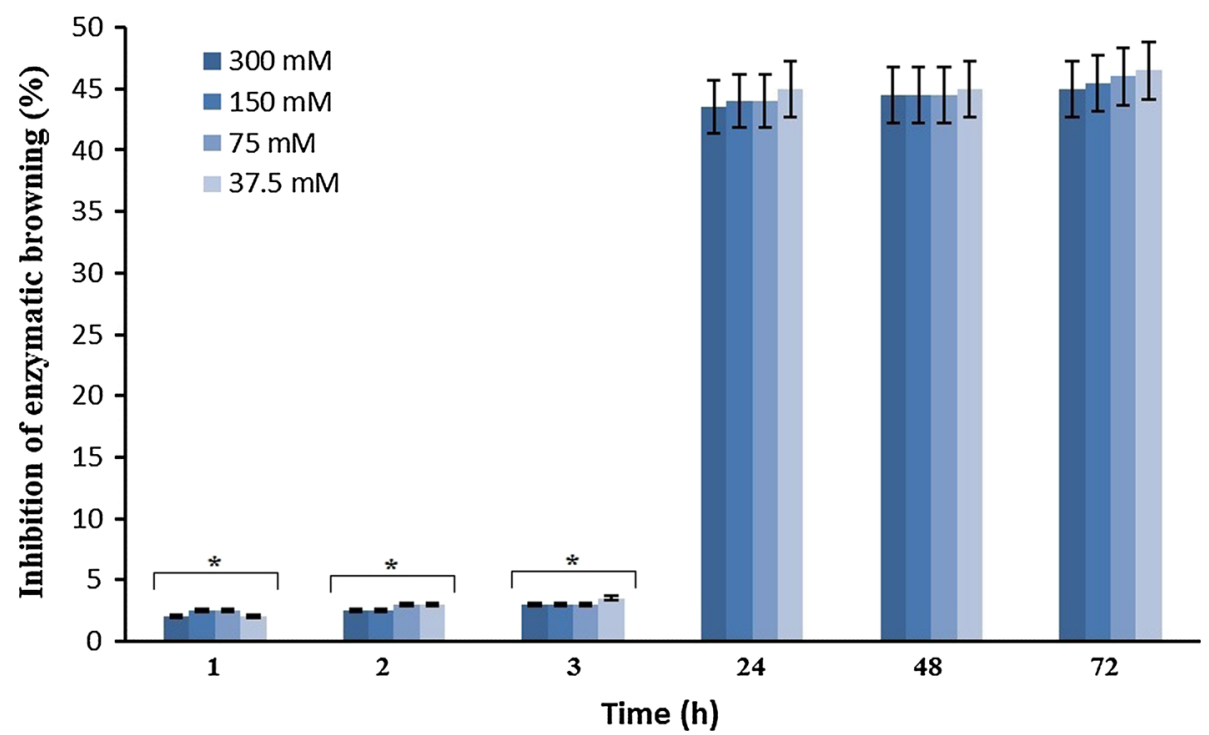

Fig. 1 The inhibition of enzymatic browning at $4{ }^{\circ} \mathrm{C} .{ }^{*} P<0.05$ compared with 24,48 , and 72 h. $n=3$

\section{Experimental study}

First, serial concentrations $(37.5,75,150$, and $300 \mathrm{mM})$ of DHPC were prepared in distilled water. In the next step, apple, banana, and mushroom were cut and separately held in petri dish. Then, $100 \mu \mathrm{L}$ of DHPC was added to all slices and incubated for $1,2,3,24,48$, and $72 \mathrm{~h}$. Temperature and $\mathrm{pH}$ were separately adjusted as follows: 


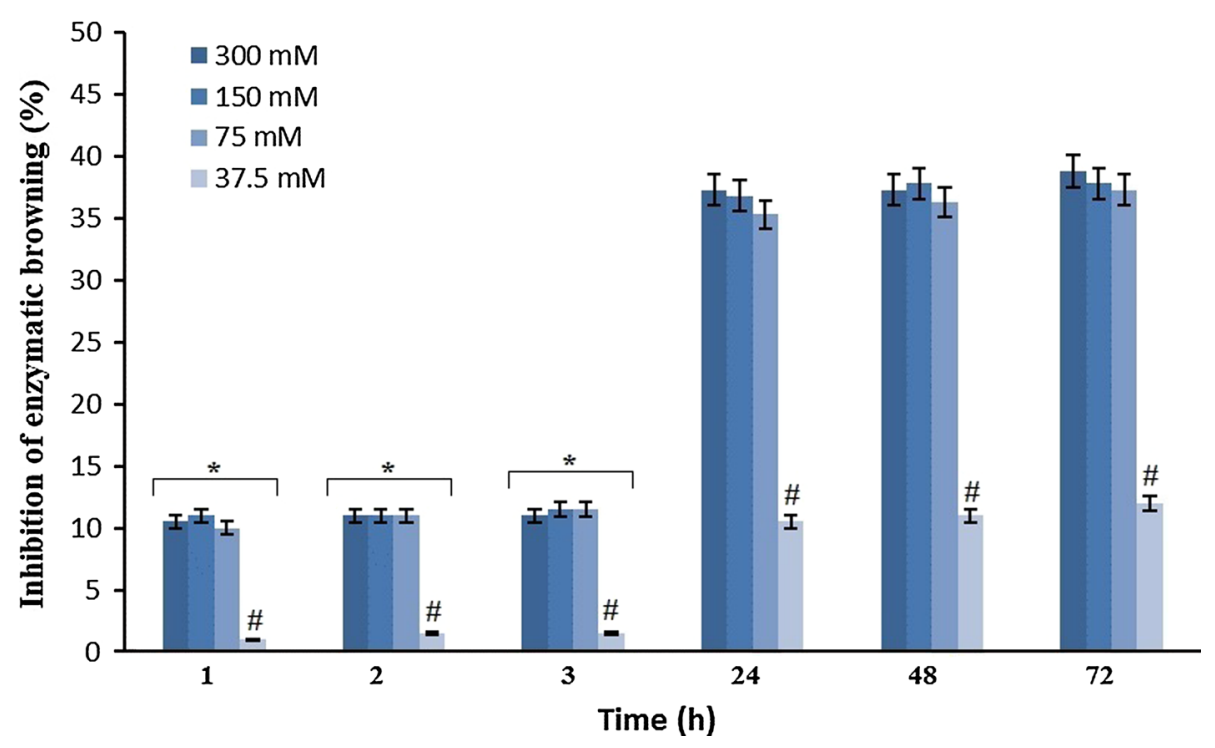

Fig. 2 The inhibition of enzymatic browning at $25^{\circ} \mathrm{C} . * P<0.05$ compared with 24,48 , and 72 h. ${ }^{\#} P<0.05$ compared with $300,150,75$ mM, $n=3$

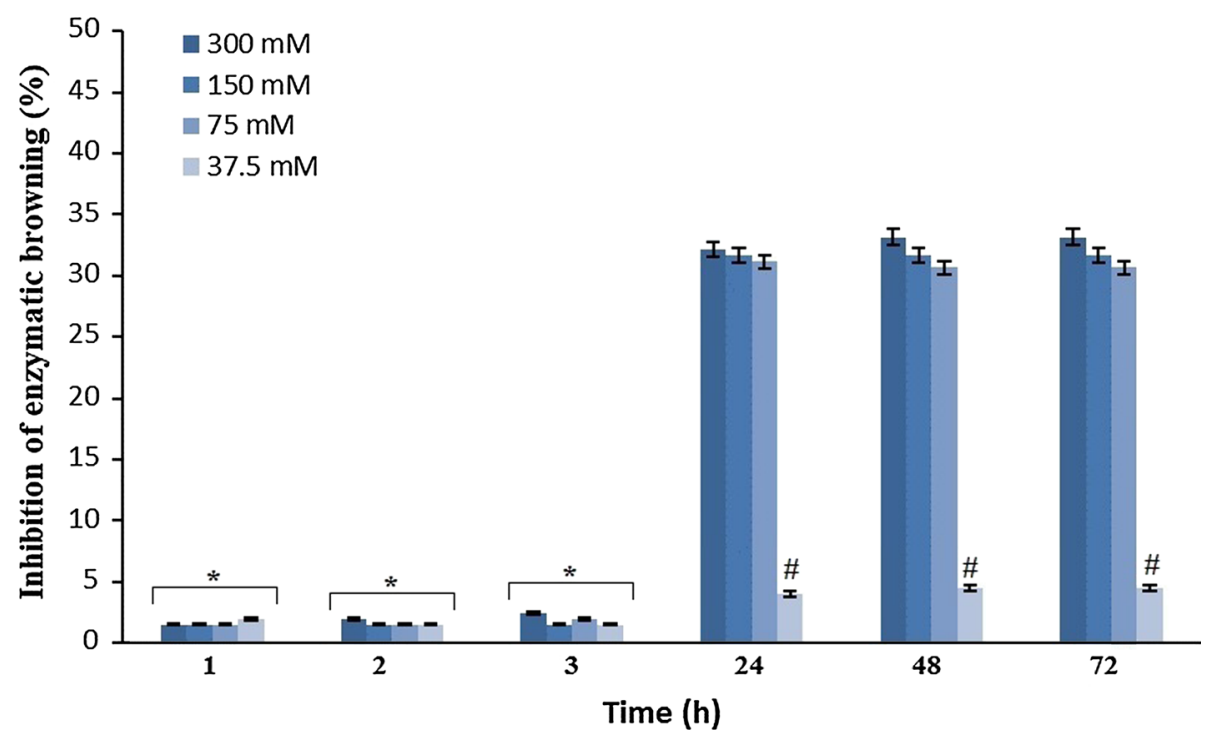

Fig. 3 The inhibition of enzymatic browning at $37^{\circ} \mathrm{C} . * P<0.05$ compared with 24,48 , and 72 h. ${ }^{*} P<0.05$ compared with $300,150,75$ mM, $n=3$

At $4{ }^{\circ} \mathrm{C}$ with $\mathrm{pH} 7$

At $25^{\circ} \mathrm{C}$ with $\mathrm{pH} 7$

At $37{ }^{\circ} \mathrm{C}$ with $\mathrm{pH} 7$

At $37^{\circ} \mathrm{C}$ with $\mathrm{pH} 5$

At $37^{\circ} \mathrm{C}$ with $\mathrm{pH} 9$

After incubation, a picture was taken from each petri dish by a digital camera with resolution of 13 mega pixel. Then, all pictures were inserted in Photoshop CS6 software, and the color of slices was recorded. The total color was measured by Formula 1. Finally, the inhibition of enzymatic browning was calculated, according to Formula 2 (Holderbaum et al. 2010).

$\sqrt{R^{2}+G^{2}+B^{2}}=$ The total color

where $R, G$, and $B$ are red, green, and blue color, respectively.

$I=\mathrm{TC}_{\mathrm{c}}-\mathrm{TC}_{\mathrm{t}} / \mathrm{TC}_{\mathrm{c}}$

where $(I)$ is the inhibition of enzymatic browning, $\mathrm{TC}_{\mathrm{c}}$ is the total color in control, and $\mathrm{TC}_{\mathrm{t}}$ is the total color in test. 

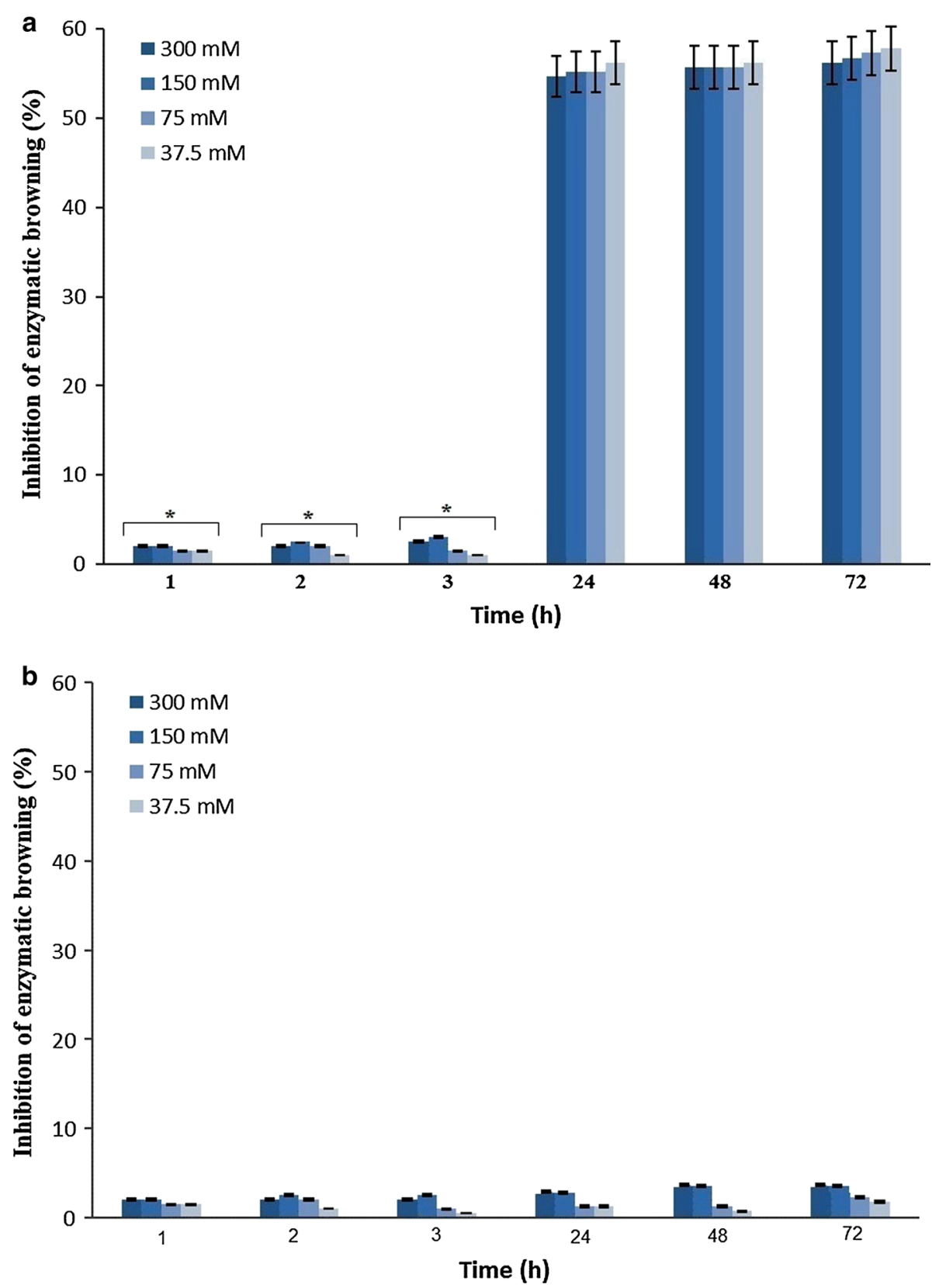

Fig. 4 The inhibition of enzymatic browning at pH 5 (a) and 9 (b). $* P<0.05$ compared with 24,48 , and 72 h, $n=3$

\section{Results and discussion}

Tables 2 and 3 show the homology between different PPOs, used in this study. As seen, the highest homology was seen between apples, and the minimum homology was observed between banana. Table 4 shows the quantity of HH, FF, HF, FH, H1-20H, F1-20F, H1-20F, and F1-20H sequences. Table 5 demonstrates the selected sequences, containing $\mathrm{H}$ and $\mathrm{F}$. Based on this result, the best sequence was histidine-leucine-phenylalanine-histidine (HLFH).
The average of intermolecular energy between HLFH and some phenolic and benzoic compounds is shown in Table 6. As seen, DHPC had less intermolecular energy.

Figures 1, 2, and 3 show the inhibition of enzymatic browning at 4,25 , and $37{ }^{\circ} \mathrm{C}$, respectively. As seen, the inhibitor had the highest efficacy at $4{ }^{\circ} \mathrm{C}$. The decrease in inhibition of enzymatic browning was seen with the increase in temperature. Figure $4 a, b$ shows the inhibition of enzymatic browning at $\mathrm{pH} 5$ and 9 , respectively. As seen, the decrease in $\mathrm{pH}$ led to increase in inhibition of enzymatic browning. 
Here, four different sequences of apple, banana, and mushroom were used. Although the GenBank number of these sequences is different, further sequences must be studied in future. The homology study showed that mushroom had less homology among others. Since mushroom is a kind of herb, its sequence has less similar to apple and banana. Since $\mathrm{H}$ and $\mathrm{F}$ are in different places of protein sequences, it cannot be certainly declared which they are in the actual place. It was found that $\mathrm{H} 1 \mathrm{H}-\mathrm{H} 2 \mathrm{H}-\mathrm{H} 3 \mathrm{H}-\mathrm{H} 4 \mathrm{H}-\mathrm{H} 5 \mathrm{H}-\mathrm{H} 8 \mathrm{H}-\mathrm{FF}-\mathrm{F} 3 \mathrm{~F}$ was similar. Remarkably, this similarity was only in number and not in sequence. Note, HLFH was the best sequence and it had the most similarity among the sequences. It must be mentioned that further studies are needed to show whether HLFH sequences are in active site or not. MD simulation showed that DHPC was the best candidate for this study. DirksHofmeister et al. (2012) compared the characteristics of PPO to find the structure-function correlation within the plant PPOs. They showed differences in enzyme-substrate interactions. Also, they found that one amino acid side chain, position $\mathrm{HB} 2+1$, was the best. Nokthai et al. (2010) analyzed the active site of PPO by molecular modeling. They found that epicatechin and catechin had high affinity with the enzyme. Based on their results, trihydroxybenzoic acid had high affinity and specificity. A homology modeling for PPO was done by Mallick et al. They showed 224 hydrogen bonds, 15 helices, and 50 turns (Mukherjee et al. 2011). Koval et al. (2006) modeled the active site of PPO. Saeidian (2013) showed the inhibition of PPO by L-glycine. The agent inhibited PPO activity at $0.4 \mathrm{mM}$ in pH 6.7. Klabunde et al. (1998) showed that the catalytic copper center was accommodated in a central four-helix bundle. Also, metal-binding sites were composed of three $\mathrm{H}$ ligands.

\section{Conclusion}

It could be concluded that DHPC can interact with PPOs. Moreover, DHPC had more efficacies at low temperature and $\mathrm{pH}$. It seems that this compound can be used for different fruits and vegetables to inhibit enzymatic browning. It must be mentioned that some additional experiments, such as toxicity test, must be done in future studies.

Acknowledgments This article was supported by Pajoohesh Medical Lab (Grant Number: 2015-9). The authors thank the laboratory staff of the Yazd Pajoohesh Medical Lab.

\section{References}

Ackaah-Gyasi NA, Zhang Y, Simpson BK (2015) Enzymes inhibitors: food and non-food impacts. Adv Food Biotechnol $1: 191-206$
Ali HM, El-Gizawy AM, El-Bassiouny RE, Saleh MA (2014) Browning inhibition mechanisms by cysteine, ascorbic acid and citric acid, and identifying PPO-catechol-cysteine reaction products. J Food Sci Technol 52:3651-3659

Ali HM, El-Gizawy AM, El-Bassiouny REI, Saleh MA (2015) The role of various amino acids in enzymatic browning process in potato tubers, and identifying the browning products. Food Chem 192:879-885

Altunkaya A, Gökmen V (2008) Effect of various inhibitors on enzymatic browning, antioxidant activity and total phenol content of fresh lettuce (Lactuca sativa). Food Chem 107:1173-1179

Artes F, Allende A (2014) Minimal processing of fresh fruit, vegetables, and juices. Food 4:121-128

Bajwa VS, Shukla MR, Sherif SM, Murch SJ, Saxena PK (2015) Identification and characterization of serotonin as an antibrowning compound of apple and pear. Postharvest Biol Technol 110:183-189

Boeckx T, Winters AL, Webb KJ, Kingston-Smith AH (2015) Polyphenol oxidase in leaves; is there any significance to the chloroplastic localization? J Exp Bot 5:141:251

Corzo-Martınez M, Corzo N, Villamiel M, del Castillo MD (2012) Browning reactions. Food Biochem Food Process 1:56-59

Dirks-Hofmeister ME, Inlow JK, Moerschbacher BM (2012) Sitedirected mutagenesis of a tetrameric dandelion polyphenol oxidase (PPO-6) reveals the site of subunit interaction. Plant Mol Biol 80:203-217

Dodd J (2014) Method for the prevention of the discoloration of fruit. Google Patents

Giri ASG (2014) Kinetics of $o$-diphenol oxidase immobilized on agarAbelmoschus esculentus polymer. Asian J Res Chem 7:919-924

Holderbaum DF, Kon T, Kudo T, Guerra MP (2010) Enzymatic browning: polyphenol oxidase activity, and polyphenols in four apple cultivars: dynamics during fruit development. HortScience 45:1150-1154

Jiménez-Atiénzar M, Cabanes J, Gandıa-Herrero F, Garcıa-Carmona F (2004) Kinetic analysis of catechin oxidation by polyphenol oxidase at neutral $\mathrm{pH}$. Biochem Biophys Res Commun 319:902-910

Klabunde T, Eicken C, Sacchettini JC, Krebs B (1998) Crystal structure of a plant catechol oxidase containing a dicopper center. Nat Struct Mol Biol 5:1084-1090

Koval IA, Gamez P, Belle C, Selmeczi K, Reedijk J (2006) Synthetic models of the active site of catechol oxidase: mechanistic studies. Chem Soc Rev 35:814-840

Kuijpers TF, Vincken J (2013) Inhibition of tyrosinase-mediated enzymatic browning by sulfite and natural alternatives. Wageningen University, Wageningen

Ma J, Sun D-W, Qu J, Liu D, Pu H, Gao W, Zeng X (2014) Applications of computer vision for assessing quality of agrifood products: a review of recent research advances. Crit Rev Food Sci Nutr 56:262-270

Mishra BB, Gautam S, Sharma A (2013) Free phenolics and polyphenol oxidase (PPO): the factors affecting post-cut browning in eggplant (Solanum melongena). Food Chem 139:105-114

Moelants K, Cardinaels R, Buggenhout S, Loey AM, Moldenaers P, Hendrickx ME (2014) A review on the relationships between processing: food structure, and rheological properties of planttissue-based food suspensions. Compr Rev Food Sci Food Saf $13: 241-260$

Mukherjee K, Mallick M, Udayakumar NA (2011) Homology modelling of polyphenol oxidase from solanum melongena: sequence analysis and structural validation studies-in silico. Int J Pharma Bio Sci 2:122-129

Niemira B, Fan X (2014) Fruits and vegetables: Advances in processing technologies to preserve and enhance the safety of 
fresh and fresh-cut fruits and vegetables. Encycl Food Microbiol 1:983-991

Nokthai P, Lee VS, Shank L (2010) Molecular modeling of peroxidase and polyphenol oxidase: substrate specificity and active site comparison International journal of molecular sciences 11:3266-3276

Quevedo R, Valencia E, Bastías JM, Cárdenas S (2014a) Description of the enzymatic browning in avocado slice using GLCM image texture. Springer, Berlin, pp 93-101

Quevedo R, Valencia E, López P, Gunckel E, Pedreschi F, Bastías J (2014b) Characterizing the variability of enzymatic browning in fresh-cut apple slices. Food Bioprocess Technol 7:1526-1532

Rico D, Martin-Diana AB, Barat J, Barry-Ryan C (2007) Extending and measuring the quality of fresh-cut fruit and vegetables: a review. Trends Food Sci Technol 18:373-386
Saeidian S (2013) Inhibition of partial purified polyphenol oxidase of Solanum lycopersicum using L-cysteine and L-glycine. Int J Basic Sci Appl Res 2:538-543

Saeidian S (2014) Effect of anti browning agents on partial purified polyphenol oxidase of hawthorn (Crataegus Spp). Int J Adv Biol Biomed Res 2:2472-2482

Wu S (2014) Glutathione suppresses the enzymatic and nonenzymatic browning in grape juice. Food Chem 160:8-10

Zhang Z et al (2015) Enzymatic browning and antioxidant activities in harvested litchi fruit as influenced by apple polyphenols. Food Chem 171:191-199

Zhou D, Li L, Wu Y, Fan J, Ouyang J (2015) Salicylic acid inhibits enzymatic browning of fresh-cut Chinese chestnut (Castanea mollissima) by competitively inhibiting polyphenol oxidase. Food Chem 171:19-25 